\title{
Strong Correlations in Electron Doped Phthalocyanine Conductors Near Half Filling
}

\author{
Erio Tosatti, ${ }^{1,2}$, 田 Michele Fabrizio, ${ }^{3,2}$ Jaroslav Tóbik, ${ }^{1}$ and Giuseppe E. Santoro ${ }^{1,2}$ \\ ${ }^{1}$ International School for Advanced Studies (SISSA), \\ and INFM Democritos National Simulation Center, Via Beirut 2-4, I-34014 Trieste, Italy \\ ${ }^{2}$ International Centre for Theoretical Physics (ICTP), P.O.Box 586, I-34014 Trieste, Italy \\ ${ }^{3}$ International School for Advanced Studies (SISSA), Via Beirut 2-4, I-34014 Trieste, Italy
}

(Dated: September 4, 2018)

\begin{abstract}
We propose that electron doped nontransition metal-phthalocyanines (MPc) like ZnPc and MgPc, similar to those very recently reported, should constitute novel strongly correlated metals. Due to orbital degeneracy, Jahn-Teller coupling and Hund's rule exchange, and with a large on-site Coulomb repulsion, these molecular conductors should display, particularly near half filling at two electrons/molecule, very unconventional properties, including Mott insulators, strongly correlated superconductivity, and other intriguing phases.
\end{abstract}

PACS numbers:

Building novel metals by doping molecular crystals such as polyacetylene, fullerenes, TTF-TCNQ, $(\mathrm{TMTSF})_{2} \mathrm{X},(\mathrm{TMTTF})_{2} \mathrm{X}$ and (BEDT-TTF $)_{2} \mathrm{X}$ salts, etc., is a well trodden route 1, 2], but remains an exciting and ever moving front. Very recently the Delft group showed that thin films of transition metal phthalocyanines (MPcs) FePc, $\mathrm{CoPc}, \mathrm{NiPc}, \mathrm{CuPc}$, initially insulating, can be turned genuinely metallic through potassium doping [3]. Electron doping appears to take place largely in the twofold degenerate lowest unoccupied $e_{g}$ molecular orbital (LUMO) [4, 5] of the molecules. The simplest rigid band model naturally explains, according to [3] , why the MPcs, initially insulating when pristine $(n=0)[\underline{6}]$, become metallic upon increasing doping $(0<n<4)$, ending up again as insulators at full doping $(n=4)$. It is not yet clear whether stoichiometric compound phases may exist here as they do in alkali doped fullerides, but there is an otherwise striking overall analogy, to the point that even the conductance values reported at optimal metallic doping are close in magnitude and temperature (in)dependence to those of $\mathrm{K}_{x} \mathrm{C}_{60}$ films.

While the pursuit of metallicity-cum-magnetism in the transition metal doped MPcs [5] will in the future constitute an interesting goal in itself, the scope of this note is to point out newer directions and possibilities that can make slightly different metallic doped MPcs, yet to be realized, potentially even more exciting. Doped MPcs are not, we claim, regular metals, but constitute strongly correlated electron systems, akin to doped two-band Mott insulators. Characterized by a strong on-site electron repulsion, by the $e_{g}$ orbital degeneracy, and by intra-site Jahn-Teller and electron-electron multiplet couplings, the doped MPc molecular system should approach near $n=2$, as foreshadowed by recent studies [7, [8, 9] a novel unstable fixed point heralding a wealth of possible low-temperature phenomena and phases. We conduct in this letter a preliminary exploration of this scenario by addressing theoretically - and thus proposing the experimental realization of - new met- als obtained through electron doping of $\mathrm{ZnPc}, \mathrm{MgPc}$ and other such non-transition metal MPcs. The extra electrons should flow into the MPc $2 e_{g}$ lowest unoccupied molecular orbital (LUMO) 4], to form a two band metal. The LUMO states are ligand shell orbitals loosely surrounding the central metal ion, with a large intramolecular Coulomb repulsion $U$ in comparison with the narrow electron bandwidth $W$ expected from weak intermolecular electron hopping. In the neighborhood of half filling, $n=2$, a doubly degenerate $e_{g}$ orbital also has a molecular Hund's rule exchange $J$ (lowering the triplet state by $4|J|$ relative to the singlet), and a Jahn-Teller (JT) coupling of the electronic state to $B_{1 g}$ and $B_{2 g} \mathrm{MPc}$ molecular vibrations, lowering the singlet state energy by an amount $E_{J T}$. Thus there will be some cancellation between the two couplings. These ingredients are similar in nature to those present in fullerides, where orders of magnitude are $U=1 \mathrm{eV}, E_{J T}=0.15 \mathrm{eV}, J=0.03 \mathrm{eV}$, and $W=0.5 \mathrm{eV}$. Although the corresponding values in MPcs are not yet so accurately known, we obtain estimates that are surprisingly close. The similarity noted in Ref. [3] between alkali doped MPcs and fullerides 2] may thus reach much deeper than just the dopability and the closeness of mobilities pointed out experimentally. As in fullerides, the resulting phase diagram will depend upon the balance between low and high spin states of the molecular ion. Unlike fullerides, however, the twofold degeneracy will reveal a potentially richer overall phase diagram.

The Hamiltonian for electrons in the $e_{g}$-orbitals of molecule $i$ is

$$
\begin{aligned}
\hat{H}_{i, m o l}= & \frac{U}{2} \hat{n}_{i}^{2}+\frac{J}{2}\left(\hat{\tau}_{i, x}^{2}+\hat{\tau}_{y, i}^{2}\right) \\
& +g\left(q_{i, 1} \hat{\tau}_{i, x}+q_{i, 2} \hat{\tau}_{i, y}\right) \\
& +\frac{\hbar \omega_{0}}{2}\left(q_{i, 1}^{2}+p_{i, 1}^{2}+q_{i, 2}^{2}+p_{i, 2}^{2}\right),
\end{aligned}
$$

where $\hat{n}_{i}$ is the overall occupation at site $i, \hat{\tau}_{i, \alpha}$, with $\alpha=x, y, z$, are pseudo-spin operators spanning the twofold degeneracy $\hat{\tau}_{i, \alpha}=\sum_{a, b=1}^{2} \sum_{\sigma} c_{i, a \sigma}^{\dagger}\left(\tau^{\alpha}\right)_{a b} c_{i, b \sigma}$, 
where $\tau^{\alpha}$ are Pauli matrices. Here $c_{i, a \sigma}^{\dagger}$ denotes creation of electrons with spin $\sigma$ at site $i$ in orbital $a=1,2$ of the LUMO $e_{g}$ doublet. The last two terms in (11) describe JT coupling of strength $g$ to a doubly degenerate vibration with frequency $\hbar \omega_{0}$, coordinates $q_{i, 1}$ and $q_{i, 2}$ and momenta $p_{i, 1}$ and $p_{i, 2}$, where $E_{J T}=8 g^{2} / \hbar \omega_{0}$. A single but degenerate mode is assumed as a simplifying approximation, replacing the actual 14 pairs of nondegenerate JT modes $B_{1 g}$ and $B_{2 g}$ allowed by the MPc $D_{4 h}$ symmetry. As detailed in note [10] an estimate of parameters appropriate to MPcs such as $\mathrm{ZnPc}, \mathrm{MgPc}$ indicates $U \sim 1 \mathrm{eV}$ and, for $n=2$, a singlet energy gain $E_{J T}-4|J| \sim 0.06-0.07 \mathrm{eV}$ over Hund's rule triplet. This prevalence of singlet over triplet, the main ingredient potentially leading to s-wave superconductivity, could be mitigated or even reversed in a solid state compound; we shall therefore examine both possibilities in the rest of this paper.

In the MPc molecular crystal, electrons can quantum mechanically hop between neighboring sites

$$
\hat{H}_{h o p}=\sum_{i j} \sum_{a, b=1}^{2} \sum_{\sigma} t_{i j}^{a b} c_{i, a \sigma}^{\dagger} c_{i, b \sigma} .
$$

Assuming first a generic three-dimensional structure with $\nu$ neighboring molecules and a weak isotropic firstneighbor hopping $t$, the LUMO $e_{g}$ orbitals will give rise to a pair of narrow molecular bands of width $W=2|t| \nu$. (We will discuss the additional signature of a quasi-onedimensional structure of these compounds in the final part of the paper.) Before doping we generally expect a narrow band $W \sim 0.3 \mathrm{eV}$ (see below), due to very weak van der Waals bonding between molecules. While $W$ will eventually depend on the intermolecular geometry, including the way that may be altered by doping, we will assume $W$ to remain of approximately the same magnitude after doping. In addition to (1) and (2) we should in principle include additional terms such as the dispersion of vibrations (to account for the coupling between the JT distortion of a molecule and those of other molecules), inter-molecular electron-electron interactions, and more. We will presently neglect them, concentrating on the basic Hamiltonian

$$
\hat{H}=\hat{H}_{h o p}+\sum_{i} \hat{H}_{i, m o l} .
$$

Because $U / W$ is large, and for $n \sim 2$, this system is generally close to a Mott transition. There, the quasiparticle band is very narrow and it is a good approximation to neglect retardation, replacing (1) with

$$
\hat{H}_{i, m o l} \rightarrow \frac{U}{2} \hat{n}_{i}^{2}+\frac{J_{e f f}}{2}\left(\hat{\tau}_{i, x}^{2}+\hat{\tau}_{y, i}^{2}\right),
$$

where $J_{e f f}=J-E_{J T} / 4$.

The further simplifying assumption of nearest- neighbor hopping that are diagonal in the orbital indices 11] brings this Hamiltonian to coincide with that recently studied by Dynamical Mean Field Theory (DMFT) 12 in Refs. 7, 81. Close to $n=2$ it was shown to display a rich phase diagram including, besides the regular metal, a Mott insulator, a strongly correlated superconductor, and a pseudo-gap metal. Within DMFT, the lattice model (3) is mapped onto an Anderson impurity self-consistently coupled to a conduction electron bath [12]. The many-body physics of the Anderson impurity and its fixed points foreshadow that of the infinite many-body system [9], as confirmed by the calculated DMFT phase diagram [7, 8]. We exploit here these results to analyse the implications for the phase diagram of half-filling doped MPcs considering both possibilities, namely: (i) singlet ground state or (ii) triplet ground state.

(i) $J_{\text {eff }}<0$ - The $n=2$ molecular ground state is a non degenerate spin singlet accompanied by a dynamical Jahn-Teller effect. If in the solid $U / W$ is larger than the critical Mott value (between 1 and 2, depending in detail on $\mathrm{J}$ and $E_{J T}$ ) the half filled MPc will realize a nonmagnetic, singlet Mott insulator [13]. For $U$ just below that value, or for light doping away from $n=2$, the MPc solid will be lightly metallic. In this "doped Mott insulator" regime the Anderson impurity displays instabilities against symmetry-broken phases in both particle-particle and particle-hole channels; which instability will eventually win out depends on band-structure and coupling details. If nesting or other band structure singularities are absent or very weak, the particle-particle instability should dominate leading to an $s$-wave superconductor with order parameter

$$
\Delta_{S C}=\left\langle c_{1 \uparrow}^{\dagger} c_{2 \downarrow}^{\dagger}+c_{2 \uparrow}^{\dagger} c_{1 \downarrow}^{\dagger}\right\rangle \neq 0 .
$$

This state is a strongly correlated superconductor (SCS) of a kind first pointed out in Ref. 14, further confirmed in the two band model in Refs. 7, 8]. It was also suggested 7, 14 - although without a calculation of $T_{c}$ - that SCS is a "high temperature superconductor" in the sense that the SCS superconducting gap may reach values several orders of magnitudes greater than the corresponding BCS value (that would be attained for $U=0), \Delta_{B C S} \sim \hbar \omega_{0} \mathrm{e}^{-1 / \lambda}$, where $\lambda=2 \rho_{0}\left|J_{e f f}\right|, \rho_{0}$ is the density of states at the Fermi level per spin and band.

If, on the contrary, the particle-hole instability channel was favored by some detail of the band structure such as Fermi surface nesting, then one of two alternative symmetry broken phases can be expected. The first is a trivial one, with order parameter

$$
\Delta_{J T}=\cos \phi\left\langle\hat{\tau}_{x}\right\rangle+\sin \phi\left\langle\hat{\tau}_{y}\right\rangle \neq 0,
$$

corresponding to a cooperative Jahn-Teller distorted 
state, either modulated or uniform, the angle $\phi$ reflecting $U(1)$ orbital symmetry breaking. The model in fact possesses $\mathrm{O}(2)$ orbital symmetry, which naturally decomposes into $\mathrm{Z}_{2} \otimes \mathrm{U}(1) . \mathrm{Z}_{2}$ is the discrete symmetry $\tau_{z} \rightarrow-\tau_{z}$, while $\mathrm{U}(1)$ represents invariance under rotations around the pseudo-spin $z$-axis. As a consequence of the static cooperative Jahn-Teller distortion, a conventional insulating band gap or at least a strong lowering of metallic density of states should take place at the Fermi level. More interesting is the alternative instability, associated with a different order parameter in the particle-hole channel

$$
\Delta_{P T}=\sum_{\alpha \beta}\left\langle c_{1 \alpha}^{\dagger} \vec{\sigma}_{\alpha \beta} \cdot \vec{S} c_{1 \beta}\right\rangle-\left\langle c_{2 \alpha}^{\dagger} \vec{\sigma}_{\alpha \beta} \cdot \vec{S} c_{2 \beta}\right\rangle \neq 0
$$

breaking both orbital $\mathrm{Z}_{2}$ and spin $\mathrm{SU}(2)$ symmetry, $\vec{S}$ being the direction along which the spin $\mathrm{SU}(2)$ symmetry is broken. We note that the LUMO $e_{g}$ orbitals are mainly localized onto the MPc four $\mathrm{N}$-atom ring and have odd parity with respect to the central metal ion. Therefore the discrete $\mathrm{Z}_{2}$ symmetry translates into parity in MPcs, whence $\Delta_{P T} \neq 0$ represents a spin current flowing on the $\mathrm{Pc}$ ring, a very intriguing possibility. This kind of phase should be either insulating or a poor metal like the cooperative JT phase. Unlike the latter, it should break both time reversal and parity, conserving only the product of the two. These properties are reminiscent of a phase discussed some time ago by Varma 15$]$.

(ii) $J_{\text {eff }}>0$ - In this case (less likely in MPcs than (i), as said above) the isolated $n=2$ molecular ion is a spin triplet. The strongest instability here is towards bulk magnetism $[\underline{9}$, ferro or antiferro depending on the band structure, a state which may or may not be accompanied by parity-symmetry breaking or by a Jahn-Teller distortion. If (depending on lattice structure) magnetism was sufficiently frustrated, then it would be possible for spintriplet superconductivity to appear with order parameter

$$
\Delta_{S C}=\left\langle c_{1 \uparrow}^{\dagger} c_{2 \downarrow}^{\dagger}-c_{2 \uparrow}^{\dagger} c_{1 \downarrow}^{\dagger}\right\rangle \neq 0
$$

Since orbitals 1 and 2 have opposite parity, this translates into a $p$-wave spin-triplet superconducting order parameter. This is an interesting possibility since, at variance with conventional $p$-wave superconductors, the pairing function is nonzero on the molecule. According to the DMFT analysis of Ref. [8], where this kind of triplet suoerconductor was first discussed, this superconducting instability is also enhanced by the proximity of a Mott insulating phase, although not as dramatically as in case (i).

Much of the uncertainty in the above scenario depends on our ignorance of the doped MPc film crystal structure, so far treated generically. A second guess based on the crystal structure of pristine MPcs suggests that the alkali doped crystals could, similar to the undoped alpha-

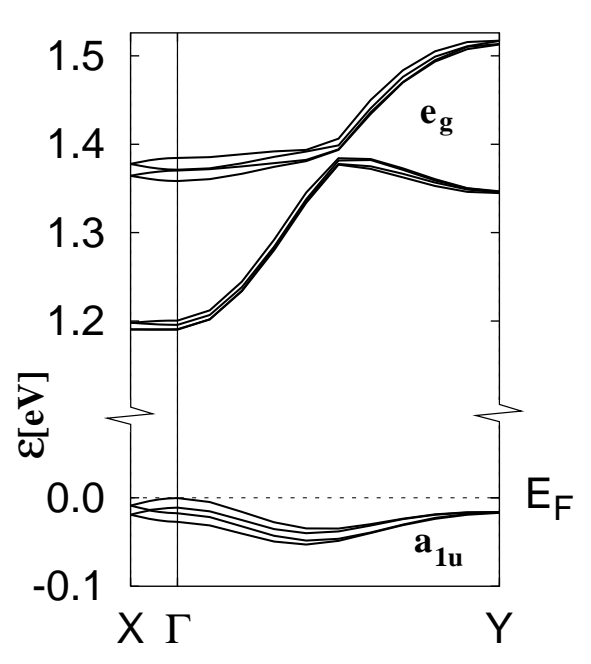

FIG. 1: Density functional electronic band structure of undoped $\alpha$ structure MgPc. The molecular stack direction is $\Gamma-Y$. The crystal structure was taken from Ref. [20]. The monoclinic cell $(a=26.29, b=3.818, c=23.92 \AA, \beta=94.6)$ contains four molecules and has symmetry $C 2 / n$.

phase of the $\mathrm{CuPc}$ films $[\underline{3}$, be made up of quasi-onedimensional (1D) chains. We carried out density functional electronic structure calculations of $\mathrm{MgPc}$ assuming an alpha phase structure with $n=0$ 16], and found an intra-chain $e_{g}$ bandwidth $W \sim 0.3 \mathrm{eV}$ but an inter-chain bandwidth between one and two orders of magnitude narrower (see Fig. . . If doped MPcs were indeed this close to 1D, then our earlier DMFT-based analysis, valid for the opposite limit of large coordination lattices, should be replaced by another where the additional effect of quantum fluctuations and other 1D specific anomalies are properly treated.

We studied this $1 \mathrm{D}$ model by adapting the two-loop renormalization group (RG) equations derived by Ref. [17] to the two-band model. The fixed point Hamiltonian towards which the model flows under RG was analyzed by means of bosonization 17, 18. At strict halffilling we found a spin-gapped insulator for any value of $U$. However, for large $U$, this was a spin-liquid Mott insulator, i.e. a spin-gapped phase without any symmetry breaking. That corresponds either to the DMFT singlet Mott state found with $J_{e f f}<0$, or to a Haldane spin-1 chain for $J_{\text {eff }}>0$. Here, the DMFT metallic phase at small $U$ is replaced by a spontaneously dimerized insulator, i.e. a spin-gapped phase with broken translational symmetry, for either sign of $J_{\text {eff }}$. Away from half-filling the model turns metallic: nonetheless we find that the spin-gap survives. In particular, in the large $U$ regime likely pertinent to the MPcs, the spin-liquid Mott insulator at $J_{\text {eff }}>0$ turns at low doping into a spin-gapped metal with dominant fluctuations in the SC singlet channel (5) and sub-dominant fluctuations in the particle-hole 
$4 k_{F}$ channels identified by Ref. [9]. The weakly-doped spin-1 chain at $J_{\text {eff }}<0$ feels on the contrary more the effects of the reduced dimensionality which causes the spin gap to survive in the metallic phase, hence preventing long-range spin-triplet superconducting correlations. Indeed, in 1D the leading SC fluctuations appear in a spin-singlet orbital-singlet but space-odd particleparticle channel [19].

Weak but nonzero interchain interactions will in a hypothetical doped MPc with alpha phase-like structure turn all these instabilities into properly long-range ordered phases at sufficiently low temperatures. While our present state of ignorance prevents further in depth discussion, the expected emerging scenario is parallel to that obtained by DMFT, apart from those differences specific to $1 \mathrm{D}$, like dimerization at $n=2$ or the persistence of a spin gap away from half-filling for regular Hund's rules.

Summarizing, we conclude first of all that stoichiometrically doped nontransition MPcs should display Mott insulating phases at all integer fillings, in particular at half filling. Analysis of a simple model (10) revealed a stunning variety of phases in the immediate neighborhood of the Mott metal-insulator transition near half filling. The variety is greater than either that predicted for the three band case fullerides 14], or that experimentally known in fullerides [2] as well as in doped organics (with lower symmetry than MPcs) near half filling [1].

Many of the phases described above will individually merit an in-depth study. At the theoretical level, approximations will need to be improved, for example by treating properly the non-degeneracy of the $B_{1 g}$ and $B_{2 g}$ molecular vibrations, the non-diagonal hopping, the dispersion of vibrations, etc. At the moment, nonetheless, it seems fair to say that more direct experimental input is urgent and essential. It will be important to dope and metallize non-transition MPcs, and to investigate whether they can be made at least locally stoichiometric. Subsequent examination by, e.g., scanning tunneling spectroscopy could search for the Mott insulating state and begin broaching in its neighborhood this fantastic scenario.

Work in SISSA/ICTP/Democritos was sponsored by MIUR FIRB RBAU017S8R004, FIRB RBAU01LX5H, and MIUR COFIN 2003, as well as by INFM. We acknowledge illuminating discussions with A. Morpurgo, A. Nersesyan, and N. Manini.

* tosatti@sissa.it

[1] See e.g.: T. Ishiguro, K. Yamaji, and G. Saito, Organic Superconductors (Springer; 2nd edition, December 1998).

[2] L. Forro and L. Mihaly, Rep. Prog. Phys. 64, 649 (2001).

[3] M.F. Craciun, S. Rogge, D.A. Wismeijer, M.J.L. den Boer, T.M. Klapwijk, and A.F. Mor- purgo, AIP Conference Proceedings 696, 489 (2003); M.F. Craciun et al., cond-mat/0401036 and to be published.

[4] M.S. Liao and S. Scheiner, J. Chem. Phys. 114, 9780 (2001).

[5] In most MPcs described in Ref. [3], there is a partly filled $d$-level. Strongly localized and with a large on-site Coulomb repulsion, that constitutes a nontrivial magnetic complication. The potentially interesting interplay of metallic conduction in the LUMO with these localized $d$ electrons is beyond the scope of this work.

[6] $\mathrm{MnPc}, \mathrm{FePc}, \mathrm{CoPc}$ and $\mathrm{CuPc}$ are open shell molecules [4], so that the pristine parent insulators must themselves constitute (single-band) Mott-Hubbard insulators.

[7] M. Capone, M. Fabrizio, C. Castellani, and E. Tosatti, cond-mat/0401090 accepted by Phys. Rev. Lett. (2004).

[8] J.E. Han, cond-mat/0401104

[9] M. Fabrizio, A.F. Ho, L. De Leo, and G.E. Santoro, Phys. Rev. Lett. 91, 246402 (2003); L. De Leo and M. Fabrizio, cond-mat/0402121 to appear in Phys. Rev. B (2004).

[10] An estimate of $U$ is given by $U=\left(E_{2}-E_{3}\right)-\left(E_{1}-E_{2}\right)=$ $A_{1}-A_{2}$, where $E_{n}$ is the total energy of the molecular ion with $n$ electrons, and $A_{n}$ its electron affinity. Calculations for isolated $\mathrm{ZnPc}(\mathrm{n}-)$ and $\mathrm{MgPc}(\mathrm{n}-)$ [4] find $A_{1} \sim 3.7 \mathrm{eV}$ and $A_{2} \sim 0.7 \mathrm{eV}$, whence $U \sim 3 \mathrm{eV}$, essentially identical to that of fullerene in vacuum[21]. In crystalline MPcs the screening could be similar to that of fullerene [22] suggesting $U=1 \div 1.5 \mathrm{eV}$ as a crude estimate. Fits of the multiplet spectra of $\mathrm{ZnPc}$ [23] suggest that in the LUMO $e_{g}$ orbitals $4|J|$ could be $\approx 0.15-0.2 \mathrm{eV}$. DFT calculations of $\mathrm{ZnPc}(2-)$ and $\mathrm{MgPc}(2-)$ yield singlet ground states, with $E_{J T}-4|J| \sim 0.06$ and $0.07 \mathrm{eV}$ respectively [4]. Resonance studies on $\mathrm{ZnPc}(2-)$ and $\operatorname{MgPc}(2-)$ in solution 24] confirms that these dianions could indeed be singlets.

[11] While the diagonal hopping approximation is not particularly realistic in the present context, it does not change the nature of the problem, and provides useful physical understanding for application to the doped MPcs.

[12] A. Georges, G. Kotliar, W. Krauth, and M.J. Rozenberg, Rev. Mod. Phys. 68, 13 (1996).

[13] M. Fabrizio and E. Tosatti, Phys. Rev. B 55, 13465 (1997).

[14] M. Capone, M. Fabrizio, C. Castellani, and E. Tosatti, Science 296, 2364 (2002).

[15] C.M. Varma, Phys. Rev. Lett. 83, 3538 (1999).

[16] J. Tóbik and E. Tosatti, unpublished (2004).

[17] M. Fabrizio, Phys. Rev. B 48, 15838 (1993).

[18] See, for instance, A.O. Gogolin, A.A. Nersesyan and A.M. Tsvelik, Bosonization and Strongly Correlated Systems (Cambridge University Press, Cambridge 1998).

[19] M. Fabrizio and A.A. Nersesyan, unpublished.

[20] C.J. Brown, J. Chem. Soc. A 1968, 2494.

[21] M. Lüders, A. Bordoni, N. Manini, A. Dal Corso, M. Fabrizio, and E. Tosatti, Philos. Mag. B 82, 1611 (2002).

[22] R.W. Lof, M.A. VanVeenendaal, B. Koopmans, H.T. Jonkman, G.A. Sawatzky, Phys. Rev. Lett. 68, 3924 (1992).

[23] P.C. Minor, M. Gouterman, and A.B.P. Lever, Inorg. Chem. 1985, 1894 (1985).

[24] D.W. Clack, N.S. Hush, and J.R. Yandle, Chem. Phys. Lett. 1, 157 (1967). 